\title{
Medical System for Internet of Things Based on WEB
}

\author{
Yuanyuan $\mathrm{Hu}^{1, \mathrm{a}^{*}}$, Xue $\mathrm{Wu}^{1, \mathrm{~b}}$ and Xining $\mathrm{Li}^{1, \mathrm{c}}$ \\ 1 Li Shi Economic and technological Development Zone Fushun City, Liaoning, P.R.China

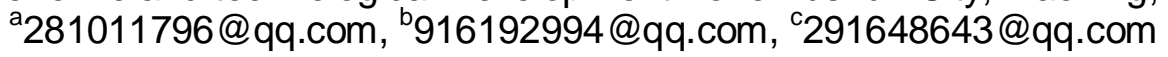

\section{Keywords: Front-end technology; HTML; Multi-terminal support}

\begin{abstract}
This system is of great significance to the image of the hospital itself and the satisfaction of the patient to the hospital window service. In general hospitals and hospitals with outpatient queuing and call number system, the efficiency of hospitals with this system is much higher than that of ordinary hospitals. The improvement of the phenomenon of "three long, one short" in patients' visits is very obvious. The characteristics of cross-platform and real-time refresh based on H5 can also make the system wide and efficient, so it is an indispensable system for hospitals, and it is also the goal that hospitals should achieve in the future.
\end{abstract}

\section{System Overall Design.}

Requirement analysis. The patient first went to the registration office to register. The general registered categories are as follows: General number, expert number, special number, special number, consultation number; The patient takes the registration form printed with the bar code and goes to the corresponding waiting area to wait. Waiting for the system software call; When the patient hears the system call their number, or when the patient sees the display to themselves, according to the display room number on the screen, take the registration form to the corresponding clinic. [1]

And a very good call system, its function design must first accord with the user's custom, simultaneously the operation is convenient, easy to learn, easy to use.The system is composed of keyboard input system, single-chip microcomputer system, screen display system and voice system. The system is divided into two parts: the control core of the pumping part and the station part is at89c51 singlechip. The singlechip is easy to program and the development tool is easy to use, which can satisfy the control data processing of queue number.

The pumping part and the station part adopt a lcd liquid crystal display, which shows the data intuitively and is easy for the customer to understand. The input of the pumping part only needs one pumping key, which is convenient for the customer to operate, and the station number part includes calling two number keys. The system adopts automatic cleaning without manual operation.

Design goal. The system consists of three parts: the server program of the system, the sub-station program of the system, and the client program (or hardware caller) of the system. The program reduces the whole process to 4 clients. Instead of the registration process, patients can use mobile devices to book registration online. The input information and the triage can be sent to the nurse for processing. Led can check the waiting situation at any time. Finally, the doctor can use the doctor to call the patient. This software improves the efficiency of seeking medical treatment and realizes the data exchange of multi-terminal at the same time. [2]

\section{Hardware System Design.}

Structure of a system. The hardware system of this program is composed of keyboard input system, single-chip microcomputer system, screen display system and call tone system. The system is divided into two parts: the pumping part and the calling number part. The control core of the system is at89c51 single chip microcomputer, which is easy to program. The development tool is easy to use and can satisfy the control data processing. [3]

After the device is powered on, the display screen is bright and no information is displayed. When the customer button, each click, the line plus 1, display customer queue number; after a number of places, then press the number button, the screen display line full. When the customer 
service call number, the team subtract 1 , the program information processing, and then display the number of the customer to the number of windows on the display, at the same time for voice prompt; when the team empty, display screen empty, The stored team number is returned to zero.

Hardware option.Mcs-51 microcontroller;1.Small and flexible, low cost, easy to produce. Can assemble all kinds of intelligent measurement and control equipment and intelligent instruments.2.Good reliability and wide application range. The microcontroller chip itself is designed according to the requirements of industrial measurement and control environment. It has strong anti-interference and can adapt to all kinds of bad environment, which is incomparable to other kinds of machines. 3. Easy to expand, easy to form a variety of application systems, strong control function. The logic control function of single chip microcomputer is very strong, the instruction system has all kinds of control function instruction, can carry on the control to the system which the logic function is quite complex. 4. With the function of communication, it can easily realize multi-computer and distributed control, and form control network and remote control. It is because 51 series single-chip computer has the above advantages, so become the processor selected for this graduation design.

At89c51 is a low voltage, high performance cmos8 bit microprocessor with $4 \mathrm{k}$ byte flicker programmable erasure read-only memory. MCU erasable read-only memory can be repeatedly erased 100 times. From the working principle, the single-chip microcomputer is a microcomputer, is a "program storage" computer. It integrates a central processor, random memory, program memory, timing / counter and various I/O interfaces on a piece of silicon chip, that is, a computer integrated on a chip.

Liquid crystal LCD1602; It is complicated to display a character with LCD because a character is composed of $6 \times 8$ or $8 \times 8$ dot matrix, and it is necessary to find 8 bytes of the display RAM region corresponding to several positions on the screen. Also make the different bits per byte 1 , the rest 0 , 1 light, 0 0. This makes up a character. But for the controller with the character generator, it is easy to display the characters. The controller can work in the text mode. According to the column number and the number of columns on the LCD, we can find the address to display the RAM, and set up the cursor. Send the code corresponding to the character here.

Hardware functional modules. By pressing the keystroke to make a registration appointment, the serial number can be sorted, and the results can be viewed through the LCD screen. When a window is free, the window button will be installed and the buzzer will ring. Prompt the patient to go to a window to see a doctor.

AT89C51 provides the following standard functions: 4k byte Flash flash memory 128-byte internal RAM, 32 I / O port lines, two 16-bit timing / counters, a 5-vector two-stage interrupt structure, a full-duplex serial communication port, an in-chip oscillator and a clock circuit. At the same time, AT89C51 can be reduced to the static logic operation of $0 \mathrm{~Hz}$, and can support two kinds of software optional power saving working mode. Idle mode stops CPU working, but allows ram, timer / counter, serial port and interrupt system to continue to work. Power down to save the contents of the RAM, but the oscillator stops working and prevents all other components from working until the next hardware reset.The pin diagram of the single chip microcomputer is shown in Figure 1. 


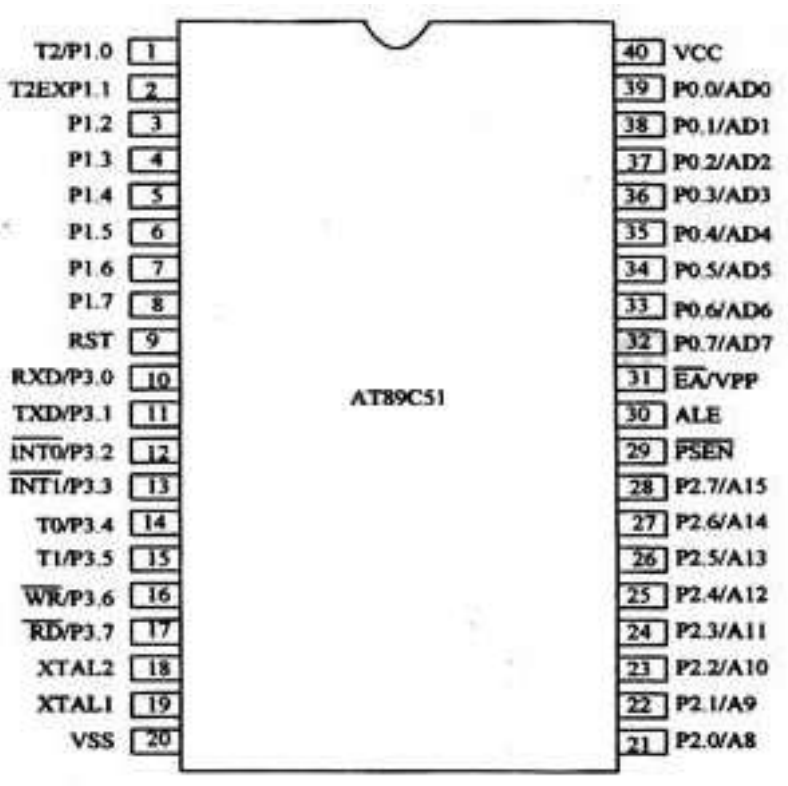

Figure1. Single chip microcomputer pin diagram

\section{Software System Design.}

Structure of a system. The language used in program development is HTML 5, the fifth major revision of the hypertext markup language introduced by the $\mathrm{W} 3 \mathrm{C}$ in 2014. Its cross-platform and real-time refresh performance brings a better user experience. WEX5 is a free open-source visual front-end development platform with better compatibility.

Client overall design. The system is divided into four programs for different groups of users: patient, nurse, doctor and LED, and the client is shown in figure 2.

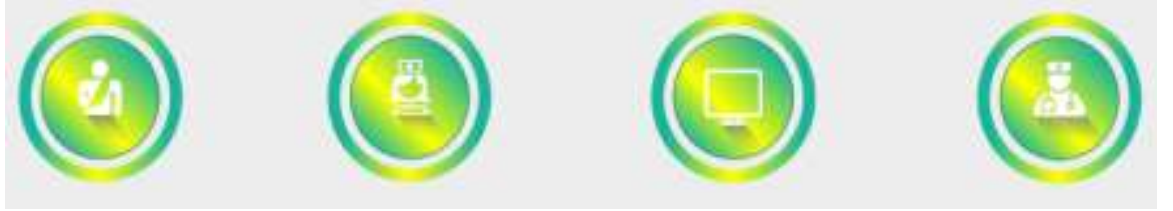

Figure 2. Client diagram

\section{Summary}

After a long period of development and final testing, it has been proved that this set of procedures can meet most of the requirements of medical treatment in the present and future medical situations, and the program based on front-end technology also shows its tenacious vitality. And widely used by the industry. And the reason it's so widely used is that it provides a whole new framework and platform for the next generation of the Internet, including features such as audio and video, and standardizes and opens these applications. So that the Internet to achieve a desktop-like experience.

\section{References}

[1] L.H. Jiang: 5G Internet of things and NB-IoT Technology (Electronic industry publishing house, China 2018),p.16(In Chinese)

[2] Francis daCosta: Reconstructing the Future of the Internet of things (Renmin University of China Press, China 2016),p.10(In Chinese)

[3] X.An: The Development of Python in the Internet of things (Electronic industry publishing house, China 2018),p.20(In Chinese) 